\title{
A Scalar Field Dark Matter Model and Its Role in the Large-Scale Structure Formation in the Universe
}

\author{
Mario A. Rodríguez-Meza \\ Departamento de Física, Instituto Nacional de Investigaciones Nucleares, Apdo. Postal 18-1027, 11801 México, DF, Mexico \\ Correspondence should be addressed to Mario A. Rodríguez-Meza, marioalberto.rodriguezmeza@gmail.com
}

Received 21 December 2011; Revised 14 March 2012; Accepted 26 March 2012

Academic Editor: Dario Nuñez

Copyright ( $) 2012$ Mario A. Rodríguez-Meza. This is an open access article distributed under the Creative Commons Attribution License, which permits unrestricted use, distribution, and reproduction in any medium, provided the original work is properly cited.

\begin{abstract}
We present a model of dark matter based on scalar-tensor theory of gravity. With this scalar field dark matter model we study the non-linear evolution of the large-scale structures in the universe. The equations that govern the evolution of the scale factor of the universe are derived together with the appropriate Newtonian equations to follow the nonlinear evolution of the structures. Results are given in terms of the power spectrum that gives quantitative information on the large-scale structure formation. The initial conditions we have used are consistent with the so-called concordance $\Lambda$ CDM model.
\end{abstract}

\section{Introduction}

The standard model of cosmology is supported by three main astronomical observations: the surveys of supernovae Ia, the cosmic microwave background radiation (CMB), and the primordial nucleosynthesis. These observations together with other modern cosmological observations, like galaxies surveys (SDSS, 2dF), galaxy rotation curves, the Bullet Cluster observation, studies of clusters of galaxies, establish that the universe behaves as dominated by dark matter (DM) and dark energy. However, the direct evidence for the existence of these invisible components remains lacking. Several theories that would modify our understanding of gravity have been proposed in order to explain the large-scale structure formation in the universe and the galactic dynamics. The best model we have to explain the observations is the $\Lambda$ CDM model, that is, the model of cold dark matter (CDM) - nonrelativistic particles of unknown origin - with cosmological constant $(\Lambda)$. In particular, this model explains very well the universe on scales of galaxy clusters and up [1].

The $\Lambda$ CDM model has become the theoretical paradigm leading the models of the universe to explain the large-scale structure (LSS) formation and several other observations, where "large" means scales larger than $1 \mathrm{Mpc}$-about the size of the group of galaxies that our Milky Way belongs to. Together with the cosmic inflation theory, this model makes a clear prediction about the necessary initial conditions that the universe has to have in order to have the structures we observe and that those structures build hierarchically due to a gravitational instability. One of its main predictions is that the density profile of galaxies, clusters of galaxies, and so on is of the form $[2,3]$

$$
\rho_{\mathrm{NFW}}(r)=\frac{\rho_{0}}{\left(r / r_{0}\right)\left(1+r / r_{0}\right)^{2}},
$$

a density profile known as Navarro-Frenk-White profile (NFW). Parameters $\rho_{0}$ and $r_{0}$ must be fitted, for example, using rotation curves of galaxies.

The $\Lambda$ CDM model and its success in explaining several observations - this is why this model is also known as the concordance model-yields the following conclusions: on large-scales, the universe is homogeneous and isotropic, as described by the Friedmann-Lamaître-Robertson-Walker (FLRW) metric. The geometry of the universe is flat, as predicted by inflation. The dark matter is cold (nonrelativistic at decoupling epoch). The initial density fluctuations were small and described by a Gaussian random field. The initial power spectrum of the density fluctuations was approximately the Harrison-Zeldovich spectrum $(P(k) \propto$ $\left.k^{n}, n=1\right)[4,5]$.

In terms of the composition of the universe, the above conclusions can be summarized as follows: Hubble's constant 
(Expansion rate of the universe at the present epoch): $H_{0}=$ $73.2 \pm 3.1 \mathrm{~km} / \mathrm{s} / \mathrm{Mpc}$. Density parameter (combined mass density of all kind of mass and energy in the universe, divided by the critical density): $\Omega_{0}=1.02 \pm 0.02$. Matter density parameter (combined mass density of all forms of matter in the universe, divided by the critical density): $\Omega_{m}=0.241 \pm$ 0.034 . Ordinary matter parameter density (density of mass of ordinary atomic matter in the universe divided by the critical density): $\Omega_{b}=0.0416 \pm 0.001$. Density parameter of dark energy (energy density of dark energy in the universe divided by the critical density): $\Omega_{\Lambda}=0.759 \pm 0.034$ [6].

Even though of all successes of the $\Lambda \mathrm{CDM}$, this model has several problems. Some of them are as follow: The exotic weakly interacting particles proposed as dark matter particles candidates are still undetected in the laboratory. The number of satellites in a galaxy such as the Milky Way is predicted to be an order of magnitude larger than is observed. The cuspy halo density profiles problem. The lack of evidence in the Milky Way for a major merger is hard to reconcile with the amount of accretion predicted by $\Lambda \mathrm{CDM}$. With respect to the inclusion of the cosmological constant, the ratio of the vacuum energy density to the radiation energy density after inflation is 1 part in $10^{100}$, a fine tuning coincidence. The cosmological constant has the wrong sign according to the string theorists who prefer a negative $\Lambda$ instead of a positive $\Lambda$. The $\Lambda \mathrm{CDM}$ model predicts that large structures should form last and therefore should be young whereas observations tell us that the largest galaxies and clusters appear old. Several candidates have been proposed in the past that pretend to substitute the role that the cosmological constant plays to accelerate the expansion of the universe. That the universe is expanding is supported mainly by the observations of the supernova project SNIa $[7,8]$. And we have led to conclude that the universe is now dominated by an energy density with negative pressure and occupies about $70 \%$ of the universe in the present epoch. This energy is called generically the dark energy. Several models to explain this dark component have been proposed, and they may be classified according to their equations of state as the follow: quintessence dark energy $[9,10]$, phantom energy $[11,12]$, or the quintom cosmology paradigm [13] (see also the review [14] for even more details). Cosmological constant has as its equation of state one in which the pressure is the negative of the density.

From the $N$-body numerical simulations point of view we have two works, in which a detailed analysis of two scalar field possibilities has been explored. One is the coupled quintessence models [15] and the other is the extended quintessence models [16]. The former considers a scalar field coupled minimally to the Ricci scalar and is coupled to the Lagrangian matter contribution. The latter is of the type of a scalar-tensor theory in which the scalar field is introduced to model dark energy with a potential that is an inverse power law. The model we present in this paper pretends to model the dark matter contribution in the large-scale structure formation with a scalar field model that stems from the newtonian limit of a general scalar-tensor theory [17].

Another problem that the $\Lambda \mathrm{CDM}$ problem is facing is the following. Almost a decade ago a bow shock in the merging cluster 1E0657-56, known as the Bullet Cluster, observed by satellite Chandra indicates that the subcluster-found by [18]-moving through this massive $\left(10^{15} h^{-1} M_{\odot}\right)$ main cluster creates a shock with a velocity as high as $4700 \mathrm{~km} \mathrm{~s}^{-1}$ $[19,20]$. A significant offset between the distribution of Xray emission and the mass distribution has been observed $[21,22]$, also indicating a high-velocity merger with gas stripped by ram pressure. Several authors have done detailed numerical noncosmological simulations [23-27]. One of the key input parameters for the simulations is to set the initial velocity of the subcluster, which is usually given at somewhere near the virial radius of the main cluster. Authors of [28] have run cosmological $N$-body simulation using a large box $\left(27 \mathrm{~h}^{-3} \mathrm{Gpc}^{3}\right)$ to calculate the distribution of infall velocities of subclusters around massive main clusters. The infall velocity distribution was given at $1-3 R_{200}$ - similar to the virial radius - and thus it gives the distribution of realistic initial velocities of subclusters just before collision. This distribution of infall velocities must be compared with the best initial velocity used by [23] of $3000 \mathrm{~km} \mathrm{~s}^{-1}$ at about $2 R_{200}$ to be in agreement with observations. Authors of [28] have found that such a high infall velocity is incompatible with the prediction of the $\Lambda \mathrm{CDM}$.

Therefore, there are plenty of problems that the concordance model has to solve and finally, it does not tell us what are dark matter and dark energy.

The program to study the large scale structure formation should be to start with primordial initial conditions which means to give the initial relevant fields, such as, for example, density and velocity fields at the epoch of last scattering $(\sim z=1100$, the value of the redshift at that epoch, i.e., a photon emitted at that epoch is redshifted as $1+z=\lambda_{r} / \lambda_{e}$, with $\lambda_{e}$ the wavelength of the photon when emitted, and $\lambda_{r}$ is the wavelength of the same photon when observed; the expansion factor for a universe with a flat geometry is related to the redshift as $1+z=1 / a)$. Then, evolve this initial condition using an $\mathrm{N}$-body scheme up to the present epoch $(z=0)$.

Some questions we have to answer are as follows What is the distribution of the LSS sizes? What is the amount of mass and its distribution at large scales? How are the voids distributed through the space? Are these voids devoid of any matter? How dose the LSS evolve with time? What is the DM equation of state? What is its role in the LSS formation processes and galactic dynamics? What are the implications of the observed LSS on the cosmological model of our universe and on the structure formation? And of course, what is the nature of the dark energy and matter?

During the last decades, there have been several proposals to explain DM, for example, Massive Compact Halo Objects (Machos), Weakly Interacting Massive Particles (WIMPs) such as supersymmetric particle like the neutralino. Other models propose that there is no dark matter and use general relativity with an appropriate equation of state. Or we can use scalar fields, minimally or nonminimally coupled to the geometry.

In this work we are mainly concerned with the problem of dark matter and its consequences on the large-scale structure formation process. Our DM model is based on using 
a scalar field (SF) that is coupled nonminimally to the metric through the Ricci scalar in the Einstein field equations. A scalar field is the most simple field of nature. Nordstrom proposed a gravity theory by 1912, before Einstein [29]. Scalar fields have been around for so many years since the pioneering work of Jordan, Brans, and Dicke. Nowadays, they are considered as (a) inflation mechanism (b) the dark matter component of galaxies (c) the quintessence field to explain dark energy and so on. Therefore, is natural to consider dark matter models based on modifications of Einstein's general relativity that include scalar fields. In this paper we will show results about the role this scalar field plays on the nonlinear large-scale structure formation of the universe. In particular, we will show how the power spectrums predicted by this model compare with the power spectrums predicted by $\Lambda \mathrm{CDM}$ and the ones that come from observations.

So we organize our work in the following form In Section 2 we present the general theory of a typical scalartensor theory (STT), that is, a theory that generalizes Einstein's general relativity by including the contribution of a scalar field that couples nonminimally to the Ricci scalar. In Section 3 we show how the Friedmann equations become within an STT and present our model for the evolution of the universe expansion factor $a(t)$. In Section 4 we present the $N$-body method which we will use to obtain the evolution of the large-scale structures. Our results for an initial condition of the fields that is consistent with the observations are given in Section 5. Finally, our conclusions are given in Section 6.

\section{General Scalar-Tensor Theory and Its Newtonian Limit}

The Lagrangian that gives us the Einstein equations of general relativity is

$$
\mathcal{L}=\frac{\sqrt{-g}}{16 \pi G} R
$$

The Einstein field equations that are obtained from the above Lagrangian, in the limit of small velocities as compared with the speed of light and small forces, limit known as the Newtonian limit, give us the standard Newtonian potential due to a point particle of mass $m$

$$
\Phi_{N}=-G \frac{m}{r}
$$

where $G$ is the gravitational constant. What we intend to do in this work is to obtain the consequences in the LSS formation processes when we rise the constant $G$ to a scalar field, $1 / G \rightarrow \phi$. But we go beyond this approach and include in the Lagrangian two additional terms that depend on this field, kinetic and potential terms. We will show, in particular, that the Newtonian limit of this theory gives for the Newtonian potential due to a mass $m[30]$,

$$
\Phi_{N}=-G \frac{m}{r}\left(1+\alpha e^{-r / \lambda}\right) .
$$

That is, the standard Newtonian potential is modified by an additional term that has the form of a Yukawa potential.
Then, we start with the Lagrangian of a general scalartensor theory

$$
\mathcal{L}=\frac{\sqrt{-g}}{16 \pi}\left[-\phi R+\frac{\omega(\phi)}{\phi}(\partial \phi)^{2}-V(\phi)\right]+\mathcal{L}_{M}\left(g_{\mu \nu}\right)
$$

Here $g_{\mu \nu}$ is the metric, $\mathcal{L}_{M}\left(g_{\mu \nu}\right)$ is the matter Lagrangian, and $\omega(\phi)$ and $V(\phi)$ are arbitrary functions of the scalar field. The fact that we have a potential term $V(\phi)$ tells us that we are dealing with a massive scalar field. Also, the first term in the brackets, $\phi R$, is the one that gives the name of nonminimally coupled scalar field.

When we make the variations of the action, $S=\int d^{4} x \mathcal{L}$, with respect to the metric and the scalar field we obtain the Einstein field equations [29]

$$
\begin{aligned}
R_{\mu \nu}-\frac{1}{2} g_{\mu \nu} R=\frac{1}{\phi} & {\left[8 \pi T_{\mu \nu} \frac{1}{2} V_{g_{\mu \nu}} \frac{\omega}{\phi} \partial_{\mu} \phi \partial_{\nu} \phi\right.} \\
& \left.-\frac{1}{2} \frac{\omega}{\phi}(\partial \phi)^{2} g_{\mu \nu}+\phi_{; \mu \nu}-g_{\mu \nu} \square \phi\right],
\end{aligned}
$$

for the metric $g_{\mu \nu}$ and for the massive SF $\phi$ we have

$$
\square \phi=\frac{1}{3+2 \omega}\left[8 \pi T-\omega^{\prime}(\partial \phi)^{2}+\phi V^{\prime}-2 V\right],
$$

where ()$^{\prime} \equiv \partial / \partial \phi$. Here $T_{\mu \nu}$ is the energy-momentum tensor with trace $T$, and $\omega(\phi)$ and $V(\phi)$ are in general arbitrary functions that govern kinetic and potential contribution of the SF. If in Lagrangian (5) we set $V(\phi)=0$ we get the Bergmann-Wagoner theory. If we further set $\omega(\phi)=$ constant the Jordan-Brans-Dicke theory is recovered. The gravitational constant is now contained in $\phi$. Also, the potential contribution, $V(\phi)$, provides mass to the SF, denoted here by $m_{\mathrm{SF}}$.

2.1. Newtonian Limit of an STT. The study of large-scale structure formation in the universe is greatly simplified by the fact that a limiting approximation of general relativity, the Newtonian mechanics, applies in a region small compared to the Hubble length $\mathrm{cH}^{-1}\left(\mathrm{cH}_{0}^{-1} \approx 3000 h^{-1} \mathrm{Mpc}\right.$, where $c$ is the speed of light, $H_{0}=100 \mathrm{~h} \mathrm{~km} / \mathrm{s} / \mathrm{Mpc}$, is Hubble's constant, and $h \approx(0.65-0.75))$ and large compared to the Schwarzschild radii of any collapsed objects. The rest of the universe affects the region only through a tidal field. The length scale $\mathrm{cH}_{0}^{-1}$ is of the order of the largest scales currently accessible in cosmological observations and $H_{0}^{-1} \approx$ $10^{10} h^{-1} \mathrm{yr}$ characterizes the evolutionary time scale of the universe [31].

Therefore, in the present study, we need to consider the influence of SF in the limit of a static STT, and then we need to describe the theory in its Newtonian approximation, that is, where gravity and the SF are weak (and time independent) and velocities of dark matter particles are nonrelativistic. We expect to have small deviations of the SF around the background field, defined here as $\langle\phi\rangle$ and can be understood 
as the scalar field beyond all matter. Accordingly we assume that the SF oscillates around the constant background field

$$
\begin{aligned}
\phi & =\langle\phi\rangle+\bar{\phi}, \\
g_{\mu \nu} & =\eta_{\mu \nu}+h_{\mu \nu},
\end{aligned}
$$

where $\eta_{\mu \nu}$ is the Minkowski metric. Then, Newtonian approximation gives [30, 32-34]

$$
\begin{aligned}
R_{00}=\frac{1}{2} \nabla^{2} h_{00} & =\frac{G_{N}}{1+\alpha} 4 \pi \rho-\frac{1}{2} \nabla^{2} \bar{\phi}, \\
\nabla^{2} \bar{\phi}-m_{\mathrm{SP}}^{2} \bar{\phi} & =-8 \pi \alpha \rho .
\end{aligned}
$$

We have set $\langle\phi\rangle=(1+\alpha) / G_{N}$ and $\alpha \equiv 1 /(3+2 \omega)$. In the above expansion we have set the cosmological constant term equal to zero, since on small galactic scales its influence should be negligible. However, at cosmological scales we do take into account the cosmological constant contribution (see below).

Note that (9) can be cast as a Poisson equation for $\psi \equiv$ $(1 / 2)\left(h_{00}+\bar{\phi} /\langle\phi\rangle\right)$

$$
\nabla^{2} \psi=4 \pi \frac{G_{N}}{1+\alpha} \rho,
$$

and the New Newtonian potential is given by $\Phi_{N} \equiv$ $(1 / 2) h_{00}=\psi-(1 / 2) \bar{\phi} /\langle\phi\rangle$. The above equation together with

$$
\nabla^{2} \bar{\phi}-\lambda^{-2} \bar{\phi}=-8 \pi \alpha \rho,
$$

forms a Poisson-Helmholtz equation and gives

$$
\Phi_{N}=\psi-\frac{1}{2} \frac{G_{N}}{1+\alpha} \bar{\phi}
$$

which represents the Newtonian limit of the STT with arbitrary potential $V(\phi)$ and function $\omega(\phi)$ that were Taylor expanded around $\langle\phi\rangle$. The resulting equations are then distinguished by the constants $G_{N}, \alpha$, and $\lambda=h_{P} / m_{\mathrm{SF}} c$. Here $h_{P}$ is Planck's constant.

The next step is to find solutions for this new Newtonian potential given a density profile, that is, to find the so-called potential density pairs. General solutions to (11) and (12) can be found in terms of the corresponding Green functions, and the new Newtonian potential is $[30,35]$

$$
\begin{aligned}
\Phi_{N}= & -\frac{G_{N}}{1+\alpha} \int \mathrm{d} \mathbf{r}_{s} \frac{\rho\left(\mathbf{r}_{s}\right)}{\left|\mathbf{r}-\mathbf{r}_{s}\right|} \\
& -\alpha \frac{G_{N}}{1+\alpha} \int \mathrm{d} \mathbf{r}_{s} \frac{\rho\left(\mathbf{r}_{s}\right) e^{-\mid \mathbf{r}-\mathbf{r}_{s} / /}}{\left|\mathbf{r}-\mathbf{r}_{s}\right|}+\text { B.C. }
\end{aligned}
$$

The First term of (14) is the contribution of the usual Newtonian gravitation (without SF), while information about the SF is contained in the second term, that is, arising from the influence function determined by the modified Helmholtz Green function, where the coupling $\omega(\alpha)$ enters as part of a source factor.

The potential of a single particle of mass $m$ can be easily obtained from (14) and is given by

$$
\Phi_{N}=-\frac{G_{N}}{1+\alpha} \frac{m}{r}\left(1+\alpha e^{-r / \lambda}\right) .
$$

For local scales, $r \ll \lambda$, deviations from the Newtonian theory are exponentially suppressed, and for $r \gg \lambda$ the Newtonian constant diminishes (augments) to $G_{N} /(1+\alpha)$ for positive (negative) $\alpha$. This means that (15) fulfills all local tests of the Newtonian dynamics, and it is only constrained by experiments or tests on scales larger than-or of the order of $-\lambda$, which in our case is of the order of galactic scales. In contrast, the potential in the form of (4) with the gravitational constant defined as usual does not fulfill the local tests of the Newtonian dynamics [36].

It is appropriate to give some additional details on the Newtonian limit for the Einstein equations without scalar fields (see [31]). We are considering a small region compared to the Hubble length $\mathrm{cH}^{-1}$ but large compared to the Schwarzschild radii of any collapsed object. In this small region the metric tensor was written as $g_{\mu \nu}=\eta_{\mu \nu}+h_{\mu \nu}$, where $h_{\mu \nu}$ is small as compared to the Minkowski metric $\eta_{\mu \nu}$. In this region Einstein's field equations are simple because the standard weak field linear approximation applies. One finds

$$
\begin{aligned}
R_{00} & =-\frac{1}{2} \eta^{\mu \nu}\left(h_{\mu \nu, 00}-h_{\mu 0, \nu 0}-h_{\nu 0, \mu 0}+h_{00, \mu \nu}\right), \\
& =\nabla_{r}^{2} \Phi, \\
g_{00} & =c^{2}+2 \Phi .
\end{aligned}
$$

Then, the zero-zero component of the field equations for an ideal fluid with density $\rho$, pressure $p$, and velocity $v \ll c$ becomes

$$
\nabla_{r}^{2} \Phi=4 \pi G_{N}\left(\rho+3 \frac{p}{c^{2}}\right)-\Lambda .
$$

For completeness the cosmological constant has been added. The geodesic equations, in the limit $v \ll c, h \ll 1$, are

$$
\frac{d^{2} r^{i}}{d t^{2}}=-\Phi_{, i}
$$

Equations (18) and (19) are the standard equations of Newtonian mechanics, except that if there is an appreciable radiation background, one must take into account the active gravitational mass associated with the pressure, and of course if $\Lambda \neq 0$, there is the cosmic force $\Lambda \mathbf{r} / 3$ between particles at separation $\mathbf{r}$.

Equations (18) and (19) apply to any observer outside a singularity, though depending on the situation, the region within which these equations apply need not contain much matter. The region can be extended by giving the observer an acceleration $g_{i}$ to bring the observer to rest relative to distant matter, which adds the term $g_{i} r^{i}$ to $\Phi$, and then by patching together the results from neighboring observers. This works (the acceleration and potentials can be added) as long as relative velocities of observers and observed matter are $\ll c$ and $\Phi \ll c^{2}$ (see (17)). For a region of size $R$ containing mass $M \sim \rho R^{3}$ with density $\rho$ roughly uniform, this second condition is

$$
G_{N} \rho R^{2} \ll c^{2} .
$$

In the Friedmann-Lemaittre models Hubble's constant is

$$
H \sim\left(G_{N} \rho\right)^{1 / 2} .
$$


If one assumes $\Lambda$ is negligible and the density parameter $\Omega \sim$ 1 , so (20) indicates

$$
R \ll c H^{-1} \sim 3000 \mathrm{Mpc} \sim 10^{28} \mathrm{~cm} .
$$

That is, the region must be small compared to the Hubble length. Since the expansion velocity is $v \approx H r$, this condition also says $v \ll c$.

The Newtonian approximation can fail at much smaller $R$ if the region includes a compact object like a neutron star or black hole, but one can deal with this by noting that at distances large compared to the Schwarzschild radius the object acts like an ordinary Newtonian point mass. It is speculated that in nuclei of galaxies there might be black holes as massive as $10^{9} M_{\odot}$, Schwarzschild radius $\sim 10^{14} \mathrm{~cm}$. If this is an upper limit, Newtonian mechanics is a good approximation over a substantial range of scales, $10^{14} \mathrm{~cm} \ll$ $r \ll 10^{28} \mathrm{~cm}$.

2.2. Multipole Expansion of the Poisson-Helmholtz Equations. The Poisson's Green function can be expanded in terms of the spherical harmonics, $Y_{l n}(\theta, \varphi)$,

$$
\frac{1}{\left|\mathbf{r}-\mathbf{r}_{s}\right|}=4 \pi \sum_{l=0}^{\infty} \sum_{n=-l}^{l} \frac{1}{2 l+1} \frac{r_{<}^{l}}{r_{>}^{l+1}} Y_{l n}^{*}\left(\theta^{\prime}, \varphi^{\prime}\right) Y_{l n}(\theta, \varphi),
$$

where $r_{<}$is the smaller of $|\mathbf{r}|$ and $\left|\mathbf{r}_{s}\right|$ and $r_{>}$is the larger of $|\mathbf{r}|$ and $\left|\mathbf{r}_{s}\right|$ and it allows us that the standard gravitational potential due to a distribution of mass $\rho(r)$, without considering the boundary condition, can be written as [37]

$$
\psi(\mathbf{r})=\psi^{(i)}+\psi^{(e)}
$$

where $\psi^{(i)}\left(\psi^{(e)}\right)$ are the internal (external) multipole expansion of $\psi$ :

$$
\begin{aligned}
\psi^{(i)} & =-\sum_{l=0}^{\infty} \sum_{n=-l}^{l} \frac{\sqrt{4 \pi}}{2 l+1} q_{l n}^{(i)} Y_{l n}(\theta, \varphi) r^{l} \\
\psi^{(e)} & =-\sum_{l=0}^{\infty} \sum_{n=-l}^{l} \frac{\sqrt{4 \pi}}{2 l+1} q_{l n}^{(e)} \frac{Y_{l n}(\theta, \varphi)}{r^{l+1}} .
\end{aligned}
$$

Here, the coefficients of the expansions $\psi^{(i)}$ and $\psi^{(e)}$, known as internal and external multipoles, respectively, are given by

$$
\begin{aligned}
& q_{l n}^{(i)}=\sqrt{4 \pi} \int_{V\left(r \leq r^{\prime}\right)} d \mathbf{r}^{\prime} \frac{1}{r^{\prime l+1}} Y_{l n}^{*}\left(\theta^{\prime}, \varphi^{\prime}\right) \rho\left(\mathbf{r}^{\prime}\right), \\
& q_{l n}^{(e)}=\sqrt{4 \pi} \int_{V\left(r>r^{\prime}\right)} d \mathbf{r}^{\prime} Y_{l n}^{*}\left(\theta^{\prime}, \varphi^{\prime}\right) r^{\prime l} \rho\left(\mathbf{r}^{\prime}\right) .
\end{aligned}
$$

The integrals are done in a region $V$ where $r \leq r^{\prime}$ for the internal multipoles and in a region $V$ where $r>r^{\prime}$ for the external multipoles. They have the property

$$
\begin{aligned}
& q_{l(-n)}^{(i)}=(-1)^{n}\left(q_{l n}^{(i)}\right)^{*}, \\
& q_{l(-n)}^{(e)}=(-1)^{n}\left(q_{l n}^{(e)}\right)^{*} .
\end{aligned}
$$

We may write expansions above in cartesian coordinates up to quadrupoles. For the internal multipole expansion we have

$$
\psi^{(i)}=-M^{(i)}-\mathbf{r} \cdot \mathbf{p}^{(i)}-\frac{1}{2} \mathbf{r} \cdot \mathbf{Q}^{(i)} \cdot \mathbf{r},
$$

and its force is

$$
\mathbf{F}_{\psi}^{(i)}=\mathbf{p}^{(i)}+\mathbf{Q}^{(i)} \cdot \mathbf{r},
$$

where

$$
\begin{aligned}
M^{(i)} & \equiv \int_{V\left(r \leq r^{\prime}\right)} d \mathbf{r}^{\prime 1 / r^{\prime}} \rho\left(\mathbf{r}^{\prime}\right), \\
p_{i}^{(i)} & \equiv \int_{V\left(r \leq r^{\prime}\right)} d \mathbf{r}^{\prime} x_{i}^{\prime} \frac{1}{r^{\prime 3}} \rho\left(\mathbf{r}^{\prime}\right), \\
Q_{i j}^{(i)} & \equiv \int_{V\left(r \leq r^{\prime}\right)} d \mathbf{r}^{\prime}\left(3 x_{i}^{\prime} x_{j}^{\prime}-r^{\prime 2} \delta_{i j}\right) \frac{1}{r^{\prime}} \rho\left(\mathbf{r}^{\prime}\right) .
\end{aligned}
$$

For the external multipoles we have

$$
\psi^{(e)}=-\frac{M^{(e)}}{r}-\frac{\mathbf{r} \cdot \mathbf{p}^{(e)}}{r^{3}}-\frac{1}{2} \frac{\mathbf{r} \cdot \mathbf{Q}^{(e)} \cdot \mathbf{r}}{r^{5}},
$$

and its force is

$$
\begin{aligned}
\mathbf{F}_{\psi}^{(e)}= & -\frac{M^{(e)}}{r^{3}} \mathbf{r}+\frac{\mathbf{p}^{(e)}}{r^{3}}-3 \frac{\mathbf{p}^{(e)} \cdot \mathbf{r}}{r^{5}} \mathbf{r} \\
& +\frac{\mathbf{Q}^{(e)} \cdot \mathbf{r}}{r^{5}}-\frac{5}{2} \frac{\mathbf{r} \cdot \mathbf{Q}^{(e)} \cdot \mathbf{r}}{r^{7}} \mathbf{r}
\end{aligned}
$$

where

$$
\begin{aligned}
M^{(e)} & \equiv \int_{V\left(r>r^{\prime}\right)} d \mathbf{r}^{\prime} \rho\left(\mathbf{r}^{\prime}\right), \\
p_{i}^{(e)} & \equiv \int_{V\left(r>r^{\prime}\right)} d \mathbf{r}^{\prime} x_{i}^{\prime} \rho\left(\mathbf{r}^{\prime}\right), \\
Q_{i j}^{(e)} & \equiv \int_{V\left(r>r^{\prime}\right)} d \mathbf{r}^{\prime}\left(3 x_{i}^{\prime} x_{j}^{\prime}-r^{\prime 2} \delta_{i j}\right) \rho\left(\mathbf{r}^{\prime}\right) .
\end{aligned}
$$

The external multipoles have the usual meaning, that is, $M^{(e)}$ is the mass, $\mathbf{p}^{(e)}$ is the dipole moment, and $\mathbf{Q}^{(e)}$ is the traceless quadrupole tensor, of the volume $V\left(r>r^{\prime}\right)$. We may attach to the internal multipoles similar meaning, that is, $M^{(i)}$ is the internal "mass," $\mathbf{p}^{(i)}$ is the internal "dipole" moment, and $\mathbf{Q}^{(i)}$ is the traceless internal "quadrupole" tensor, of the volume $V\left(r \leq r^{\prime}\right)$.

In the case of the scalar field, with the expansion

$$
\begin{aligned}
& \frac{\exp \left(-m\left|\mathbf{r}-\mathbf{r}_{s}\right|\right)}{\left|\mathbf{r}-\mathbf{r}_{s}\right|} \\
& =4 \pi m \sum_{l=0}^{\infty} \sum_{n=-l}^{l} i_{l}\left(m r_{<}\right) k_{l}\left(m r_{>}\right) Y_{l n}^{*}\left(\theta^{\prime}, \varphi^{\prime}\right) Y_{l n}(\theta, \varphi),
\end{aligned}
$$

the contribution of the scalar field to the Newtonian gravitational potential can be written as

$$
\bar{\phi}(\mathbf{r})=\bar{\phi}^{(i)}+\bar{\phi}^{(e)}
$$


where, for simplicity of notation, we are using $m=m_{\mathrm{SF}}=$ $h_{P} /(c \lambda)$ and

$$
\begin{aligned}
\frac{1}{2 \alpha} \bar{\phi}^{(i)} & =\sqrt{4 \pi} \sum_{l=0}^{\infty} \sum_{n=-l}^{l} \frac{i_{l}(m r)}{(m r)^{l}} \bar{q}_{l n}^{(i)} r^{l} Y_{l n}(\theta, \varphi), \\
\frac{1}{2 \alpha} \bar{\phi}^{(e)} & =\sqrt{4 \pi} \sum_{l=0}^{\infty} \sum_{n=-l}^{l}(m r)^{l+1} k_{l}(m r) \bar{q}_{l n}^{(e)} \frac{Y_{l n}(\theta, \varphi)}{r^{l+1}} .
\end{aligned}
$$

$i_{l}(x)$ and $k_{l}(x)$ are the modified spherical Bessel functions.

We have defined the multipoles for the scalar field as

$$
\begin{aligned}
& \bar{q}_{l n}^{(i)}=\sqrt{4 \pi} \int_{V\left(r \leq r^{\prime}\right)} d \mathbf{r}^{\prime} \frac{Y_{l n}^{*}\left(\theta^{\prime}, \varphi^{\prime}\right)}{r^{\prime l+1}}\left(m r^{\prime}\right)^{l+1} k_{l}\left(m r^{\prime}\right) \rho\left(\mathbf{r}^{\prime}\right), \\
& \bar{q}_{l n}^{(e)}=\sqrt{4 \pi} \int_{V\left(r>r^{\prime}\right)} d \mathbf{r}^{\prime} Y_{l n}^{*}\left(\theta^{\prime}, \varphi^{\prime}\right) \frac{i_{l}\left(m r^{\prime}\right)}{\left(m r^{\prime}\right)^{l}} r^{\prime l} \rho\left(\mathbf{r}^{\prime}\right) .
\end{aligned}
$$

They, also, have the property

$$
\begin{aligned}
& \bar{q}_{l(-n)}^{(i)}=(-1)^{n}\left(\bar{q}_{l n}^{(i)}\right)^{*}, \\
& \bar{q}_{l(-n)}^{(e)}=(-1)^{n}\left(\bar{q}_{l n}^{(e)}\right)^{*} .
\end{aligned}
$$

The above expansions of SF contribution to the Newtonian potential can be written in cartesian coordinates. The internal multipole expansion of the SF contribution, up to quadrupoles, is

$$
\begin{aligned}
\frac{1}{2 \alpha} \bar{\phi}^{(i)}= & i_{0}(m r) \bar{M}^{(i)}+3 \frac{i_{1}(m r)}{m r} \mathbf{r} \cdot \overline{\mathbf{p}}^{(i)} \\
& +5 \frac{1}{2} \frac{i_{2}(m r)}{(m r)^{2}} \mathbf{r} \cdot \overline{\mathbf{Q}}^{(i)} \cdot \mathbf{r}
\end{aligned}
$$

and its force is

$$
\begin{aligned}
\frac{1}{2 \alpha} \mathbf{F}_{\phi}^{(i)}= & -m^{2} \frac{i_{1}(m r)}{m r} \bar{M}^{(i)} \mathbf{r}-3 \frac{i_{1}(m r)}{m r} \overline{\mathbf{p}}^{(i)} \\
& -3 m^{2} \frac{i_{2}(m r)}{(m r)^{2}}\left(\overline{\mathbf{p}}^{(i)} \cdot \mathbf{r}\right) \mathbf{r}-5 \frac{i_{2}(m r)}{(m r)^{2}} \overline{\mathbf{Q}}^{(i)} \cdot \mathbf{r} \\
& +5 \frac{1}{2 r^{2}}\left[5 \frac{i_{2}(m r)}{(m r)^{2}}-\frac{i_{1}(m r)}{m r}\right]\left(\mathbf{r} \cdot \overline{\mathbf{Q}}^{(i)} \cdot \mathbf{r}\right) \mathbf{r}
\end{aligned}
$$

where

$$
\begin{aligned}
\bar{M}^{(i)} & \equiv \int_{V\left(r \leq r^{\prime}\right)} d \mathbf{r}^{\prime}\left(m r^{\prime}\right) k_{0}\left(m r^{\prime}\right) \frac{1}{r^{\prime}} \rho\left(\mathbf{r}^{\prime}\right), \\
\bar{p}_{i}^{(i)} & \equiv \int_{V\left(r \leq r^{\prime}\right)} d \mathbf{r}^{\prime}\left(m r^{\prime}\right)^{2} k_{1}\left(m r^{\prime}\right) x_{i}^{\prime} \frac{1}{r^{\prime 3}} \rho\left(\mathbf{r}^{\prime}\right), \\
\bar{Q}_{i j}^{(i)} & \equiv \int_{V\left(r \leq r^{\prime}\right)} d \mathbf{r}^{\prime}\left(m r^{\prime}\right)^{3} k_{2}\left(m r^{\prime}\right)\left(3 x_{i}^{\prime} x_{j}^{\prime}-r^{\prime 2} \delta_{i j}\right) \frac{1}{r^{\prime 5}} \rho\left(\mathbf{r}^{\prime}\right) .
\end{aligned}
$$

In the exterior region the SF multipole contribution to the potential is

$$
\begin{aligned}
\frac{1}{2 \alpha} \bar{\phi}^{(e)}= & m r k_{0}(m r) \frac{\bar{M}^{(e)}}{r}+3(m r)^{2} k_{1}(m r) \frac{\mathbf{r} \cdot \overline{\mathbf{p}}^{(e)}}{r^{3}} \\
& +5 \frac{1}{2}(m r)^{3} k_{2}(m r) \frac{\mathbf{r} \cdot \overline{\mathbf{Q}}^{(e)} \cdot \mathbf{r}}{r^{5}}
\end{aligned}
$$

and its force is

$$
\begin{aligned}
\frac{1}{2 \alpha} \mathbf{F}_{\phi}^{(e)}= & (m r)^{2} k_{1}(m r) \frac{\bar{M}^{(e)}}{r^{3}} \mathbf{r}-3(m r)^{2} k_{1}(m r) \frac{\overline{\mathbf{p}}^{(e)}}{r^{3}} \\
+ & 3\left[(m r) k_{0}(m r)\right. \\
& \left.+(2+m r)(m r)^{2} k_{1}(m r)\right] \frac{\left(\overline{\mathbf{p}}^{(e)} \cdot \mathbf{r}\right)}{r^{5}} \mathbf{r} \\
- & 5(m r)^{3} k_{2}(m r) \frac{\overline{\mathbf{Q}}^{(e)} \cdot \mathbf{r}}{r^{5}} \\
+ & \frac{5}{2}\left[3(m r) k_{0}(m r)+3(m r)^{2} k_{1}(m r)\right. \\
& \left.+(3+m r)(m r)^{3} k_{2}(m r)\right] \frac{\left(\mathbf{r} \cdot \overline{\mathbf{Q}}^{(e)} \cdot \mathbf{r}\right)}{r^{7}} \mathbf{r},
\end{aligned}
$$

where

$$
\begin{aligned}
\bar{M}^{(e)} & \equiv \int_{V\left(r>r^{\prime}\right)} d \mathbf{r}^{\prime} i_{0}\left(m r^{\prime}\right) \rho\left(\mathbf{r}^{\prime}\right), \\
\bar{p}_{i}^{(e)} & \equiv \int_{V\left(r>r^{\prime}\right)} d \mathbf{r}^{\prime} \frac{i_{1}\left(m r^{\prime}\right)}{m r^{\prime}} x_{i}^{\prime} \rho\left(\mathbf{r}^{\prime}\right), \\
\bar{Q}_{i j}^{(e)} & \equiv \int_{V\left(r>r^{\prime}\right)} d \mathbf{r}^{\prime} \frac{i_{2}\left(m r^{\prime}\right)}{\left(m r^{\prime}\right)^{2}}\left(3 x_{i}^{\prime} x_{j}^{\prime}-r^{\prime 2} \delta_{i j}\right) \rho\left(\mathbf{r}^{\prime}\right) .
\end{aligned}
$$

In the limit when $m \rightarrow 0$ we recover the standard Newtonian potential and force expressions.

Up to here the formulation is general; that is, mass distribution may have any symmetry or none at all. In order to take advantage of the symmetry of the spherical harmonics, the mass distribution must be spherically symmetric.

\section{Cosmological Evolution Equations Using a Static STT}

To simulate cosmological systems, the expansion of the universe has to be taken into account. Also, to determine the nature of the cosmological model we need to determine the composition of the universe, that is, we need to give the values of $\Omega_{i} \equiv \rho_{i} / \rho_{c}$, with $\rho_{c}=3 H^{2} / 8 \pi G_{N}$, for each component $i$, taking into account in this way all forms of energy densities that exist at present. If a particular kind of energy density is described by an equation of state of the form $p=w \rho$, where $p$ is the pressure and $w$ is a constant, then the equation for energy conservation in an expanding background, $d\left(\rho a^{3}\right)=-p d\left(a^{3}\right)$, can be integrated to give $\rho \propto a^{-3(1+w)}$.

Then, the Friedmann equation for the expansion factor $a(t)$ is written as

$$
\frac{\dot{a}^{2}}{a^{2}}=H_{0}^{2} \sum_{i} \Omega_{i}\left(\frac{a_{0}}{a}\right)^{3\left(1+w_{i}\right)}-\frac{k}{a^{2}},
$$

where $w_{i}$ characterizes equation of state of species $i$.

The most familiar forms of energy densities are those due to pressureless matter with $w_{i}=0$ (i.e., nonrelativistic matter with rest-mass-energy density $\rho c^{2}$ dominating over the kinetic energy density $\rho v^{2} / 2$ ) and radiation with $w_{i}=1 / 3$. 
The density parameter contributed today by visible, nonrelativistic, baryonic matter in the universe is $\Omega_{B} \approx(0.04-0.05)$ and the density parameter that is due to radiation is $\Omega_{R} \approx$ $2 \times 10^{-5}$.

In this work we will consider a model with only two energy density contributions. One is a pressureless and nonbaryonic dark matter with $\Omega_{\mathrm{DM}} \approx 0.3$ that does not couple with radiation, although in the numerical simulations we may include in $\Omega_{\mathrm{DM}}$ the baryonic matter. The other will be a cosmological constant contribution $\Omega_{\Lambda} \approx 0.7$ with and equation of state $p=-\rho$. The above equation for $a(t)$ becomes

$$
\frac{\dot{a}^{2}}{a^{2}}=H_{0}^{2}\left[\Omega_{\mathrm{DM}}\left(\frac{a_{0}}{a}\right)^{3}+\Omega_{\Lambda}\right]-\frac{k}{a^{2}} .
$$

The above discussion gives us the standard cosmological model with cosmological constant, that is, $\Lambda \mathrm{CDM}$ model.

In the framework of a scalar-tensor theory the cosmology is given as follows. If we use the Friedmann metric [29]

$$
d s^{2}=-d t^{2}+a^{2}(t)\left[\frac{d r^{2}}{1-\kappa r^{2}}+r^{2}\left(d \theta^{2}+\sin ^{2} \theta d \varphi^{2}\right)\right]
$$

in the time-time component of the Einstein field equations (Hamiltonian constraint) it gives

$$
H^{2}=\frac{8 \pi}{3 \phi} \rho-H \frac{\dot{\phi}}{\phi}+\frac{\omega(\phi)}{6}\left(\frac{\dot{\phi}}{\phi}\right)^{2}-\frac{\kappa}{a^{2}}+\frac{V}{6 \phi}
$$

while the equation for the scalar field is

$$
\begin{aligned}
\ddot{\phi}+\left(3 H+\frac{\dot{\omega}}{2 \omega+3}\right) \dot{\phi} \\
\quad=\frac{1}{2 \omega+3}\left[8 \pi(\rho-3 P)-\phi \frac{d V}{d \phi}+2 V\right]
\end{aligned}
$$

and the equation of the fluid is

$$
\dot{\rho}+3 H(\rho+P)=0 .
$$

The cosmological evolution of the initial perturbed fields should be computed using the above equations for the expansion factor. However, here, we will employ a cosmological model with a static SF which is consistent with the Newtonian limit given above. Thus, the scale factor, $a(t)$, is given by the following Friedman model:

$$
a^{3} H^{2}=H_{0}^{2}\left[\frac{\Omega_{\mathrm{DM} 0}+\Omega_{\Lambda 0} a^{3}}{1+\alpha}+\left(1-\frac{\Omega_{\mathrm{DM} 0}+\Omega_{\Lambda 0}}{1+\alpha}\right) a\right],
$$

where $H=\dot{a} / a$ and $\Omega_{\mathrm{DM} 0}$ and $\Omega_{\Lambda 0}$ are the matter and energy density evaluated at present, respectively. The denominator $(1+\alpha)$ appears in $(51)$ due to the fact that we have defined $\Omega_{i} \equiv \rho / \rho_{c}$ and $\rho_{c}=3 H^{2} / 8 \pi G_{N}$. Then, whenever appears the gravitational constant $G_{N}$, we replace it by $G_{N} /(1+\alpha)$.

We notice that the source of the cosmic evolution is deviated by the term $1+\alpha$ when compared to the standard

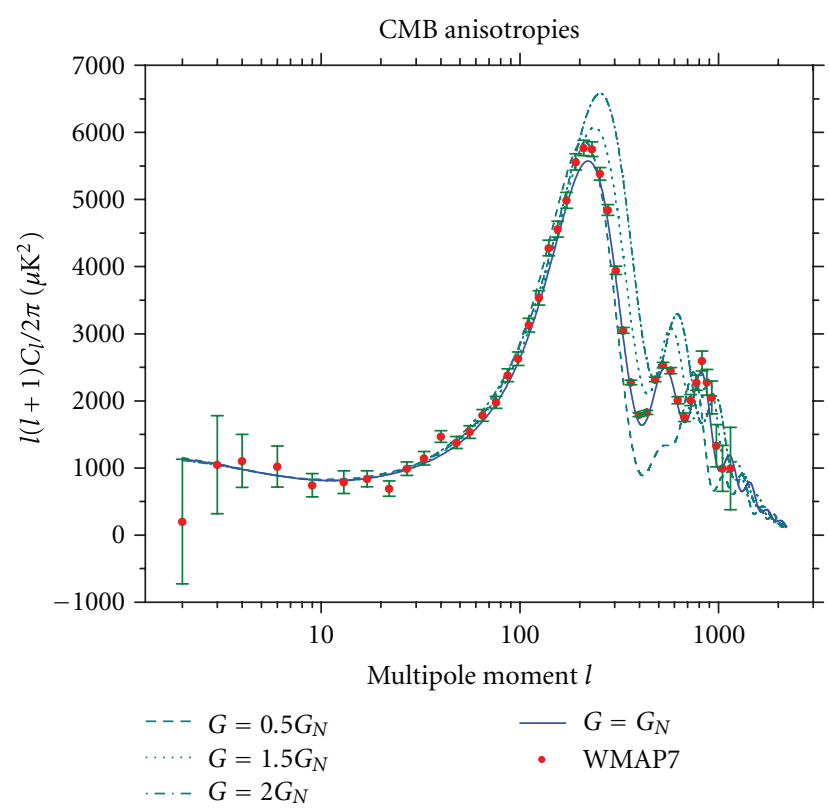

FIGURE 1: Cosmic microwave background anisotropies. With small dash symbol are the WMAP 7 years experimental observations [53]. We have plotted several cases in which the Newton constant has been modified. The standard case is shown with a small dash line. With a long dash line is shown an effective gravitational constant, $G_{\text {eff }}=0.5 G_{N}$. With a dotted line is shown the case with $G_{\text {eff }}=$ $1.5 G_{N}$, and with a dash-dotted line is shown the case with $G_{\text {eff }}=$ $2 G_{N}$.

Friedmann-Lemaitre model. Therefore, it is convenient to define a new density parameter by $\Omega_{i}^{(\alpha)} \equiv \Omega_{i} /(1+\alpha)$. This new density parameter is such that $\Omega_{\mathrm{DM}}^{(\alpha)}+\Omega_{\Lambda}^{(\alpha)}=1$, which implies a flat universe, and this shall be assumed in the following computations, where we consider $\left(\Omega_{m}^{(\alpha)}, \Omega_{\Lambda}^{(\alpha)}\right)=$ $(0.3,0.7)$.

For positive values of $\alpha$, a flat cosmological model demands to have a factor $(1+\alpha)$ with more energy content $\left(\Omega_{m}\right.$ and $\left.\Omega_{\Lambda}\right)$ than in standard cosmology. On the other hand, for negative values of $\alpha$ one needs a factor $(1+\alpha)$ with less $\Omega_{m}$ and $\Omega_{\Lambda}$ to have a flat universe. To be consistent with the CMB spectrum and structure formation numerical experiments, cosmological constraints must be applied on $\alpha$ in order for it to be within the range $(-1,1)$ [38-41]. In Figure 1 we show the effect of different values of the gravitational constant $G_{N}$ on the anisotropies of the CMB.

\section{Vlasov-Poisson-Helmholtz Equations and the $N$-Body Method}

The Vlasov-Poisson equation in an expanding universe describes the evolution of the six-dimensional, one-particle distribution function, $f(\mathbf{x}, \mathbf{p})$. The Vlasov equation is given by

$$
\frac{\partial f}{\partial t}+\frac{\mathbf{p}}{m a^{2}} \cdot \frac{\partial f}{\partial \mathbf{x}}-m \nabla \Phi_{N}(\mathbf{x}) \cdot \frac{\partial f}{\partial \mathbf{p}}=0,
$$


where $\mathbf{x}$ is the comoving coordinate, $\mathbf{p}=m a^{2} \dot{\mathbf{x}}, m$ is the particle mass, and $\Phi_{N}$ is the self-consistent gravitational potential given by the Poisson equation,

$$
\nabla^{2} \Phi_{N}(\mathbf{x})=4 \pi G_{N} a^{2}\left[\rho(\mathbf{x})-\rho_{b}(t)\right],
$$

where $\rho_{b}$ is the background mass density. The Vlasov-Poisson system, (56) and (53) form the Vlasov-Poisson equation, constitutes a collisionless, mean-field approximation to the evolution of the full $\mathrm{N}$-body distribution.

An $\mathrm{N}$-body code attempts to solve the Vlasov-Poisson system of equations by representing the one-particle distribution function as

$$
f(\mathbf{x}, \mathbf{p})=\sum_{i=1}^{N} \delta\left(\mathbf{x}-\mathbf{x}_{i}\right) \delta\left(\mathbf{p}-\mathbf{p}_{i}\right) .
$$

Substitution of (54) in the Vlasov-Poisson system of equations yields the exact Newton's equations for a system of $N$ gravitating particles (see [42] for details):

$$
\ddot{\mathbf{x}}_{i}+2 H \mathbf{x}_{i}=-\frac{1}{a^{3}} G_{N} \sum_{j \neq i} \frac{m_{j}\left(\mathbf{x}_{i}-\mathbf{x}_{j}\right)}{\left|\mathbf{x}_{i}-\mathbf{x}_{j}\right|^{3}},
$$

where the sum includes all periodic images of particle $j$ and numerically is done using the Ewald method (see [43]). It is important to keep in mind, however, that we are not really interested in solving the exact $N$-body problem for a finite number of particles, $N$. The actual problem of interest is the exact $N$-body problem in the fluid limit, that is, as $N \rightarrow$ $\infty$. For this reason, one important aspect of the numerical fidelity of $\mathrm{N}$-body codes lies in controlling errors due to the discrete nature of the particle representation of $f(\mathbf{r}, \mathbf{p})$.

In the Newtonian limit of STT of gravity to describe the evolution of the six-dimensional, one-particle distribution function, $f(\mathbf{x}, \mathbf{p}, t)$, we need to solve the Vlasov-PoissonHelmholtz equation in an expanding universe [44-48]. The Vlasov equation is given by

$$
\frac{\partial f}{\partial t}+\frac{\mathbf{p}}{m a^{2}} \cdot \frac{\partial f}{\partial \mathbf{x}}-m \nabla \Phi_{N}(\mathbf{x}) \cdot \frac{\partial f}{\partial \mathbf{p}}=0
$$

where $\Phi_{N}=(1 / 2) h_{00}=\psi-(1 / 2)\left(G_{N} /(1+\alpha)\right) \bar{\phi}$ with $\psi$ satisfying Poisson equation

$$
\nabla^{2} \psi(\mathbf{x})=4 \pi G_{N} a^{2}\left[\rho(\mathbf{x})-\rho_{b}(t)\right]
$$

and $\bar{\phi}$ satisfying the Helmholtz equation

$$
\nabla^{2} \bar{\phi}-\lambda^{-2} \bar{\phi}=-8 \pi \alpha a^{2}\left[\rho(\mathbf{x})-\rho_{b}(t)\right] .
$$

The above equations form the Vlasov-Poisson-Helmholtz system of equations that constitutes a collisionless, meanfield approximation to the evolution of the full $\mathrm{N}$-body distribution in the framework of the Newtonian limit of a scalar-tensor theory.

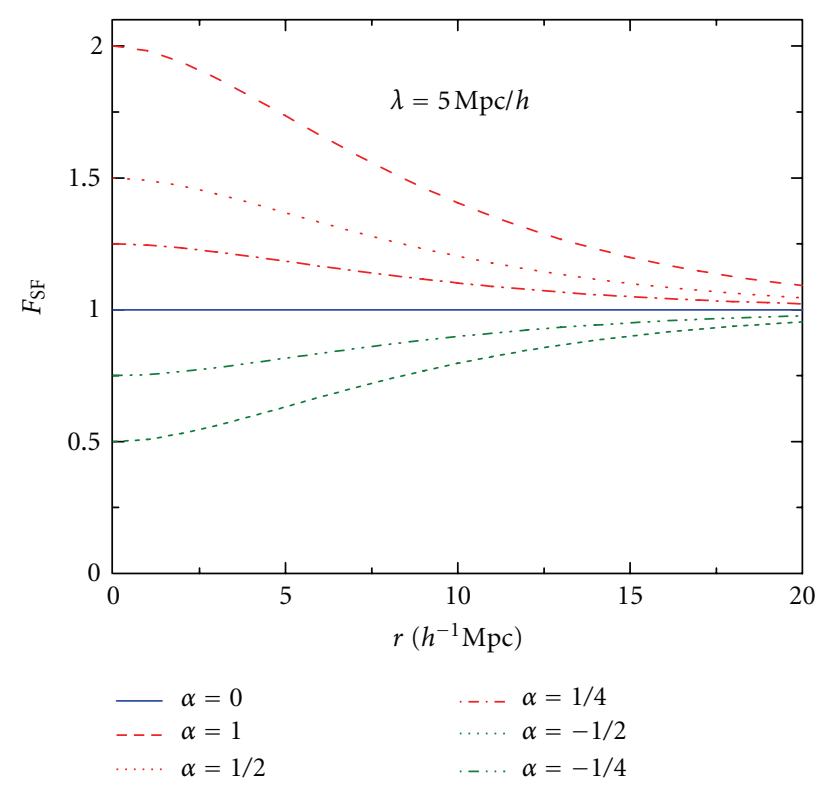

FIGURE 2: Function $F_{\mathrm{SF}}(r, \alpha, \lambda)$ for $\lambda=5 \mathrm{Mpc} / h$ and several values of parameter $\alpha . \alpha=0$, That is, the standard Newtonian case is the horizontal line. The long dashed line above the horizontal standard line is for $\alpha=1$. The dotted line is for $\alpha=1 / 2$, and the dotteddashed line is for $\alpha=1 / 4$. For negative values of $\alpha$ the lines are below the standard horizontal case. Small dashed line is for $\alpha=$ $-1 / 2$ and the double-dotted-dashed line is for $\alpha=-1 / 4$.

Using the representation of the one-particle distribution function (54) the Newtonian motion equation for a particle $i$ is written as [45]

$$
\begin{aligned}
\ddot{\mathbf{x}}_{i}+2 H \mathbf{x}_{i}= & -\frac{1}{a^{3}} \frac{G_{N}}{1+\alpha} \sum_{j \neq i} \frac{m_{j}\left(\mathbf{x}_{i}-\mathbf{x}_{j}\right)}{\left|\mathbf{x}_{i}-\mathbf{x}_{j}\right|^{3}} \\
& \times F_{\mathrm{SF}}\left(\left|\mathbf{x}_{i}-\mathbf{x}_{j}\right|, \alpha, \lambda\right),
\end{aligned}
$$

where the sum includes all periodic images of particle $j$, and $F_{\mathrm{SF}}(r, \alpha, \lambda)$ is

$$
F_{\mathrm{SF}}(r, \alpha, \lambda)=1+\alpha\left(1+\frac{r}{\lambda}\right) e^{-r / \lambda}
$$

which, for small distances compared to $\lambda$, is $F_{\mathrm{SF}}(r<\lambda, \alpha, \lambda) \approx$ $1+\alpha(1+r / \lambda)$ and, for long distances, is $F_{\mathrm{SF}}(r>\lambda, \alpha, \lambda) \approx 1$, as in Newtonian physics.

The function $F_{\mathrm{SF}}(r, \alpha, \lambda)$ is shown in Figure 2 for several values of parameter $\alpha$. The horizontal line at $F_{\mathrm{SF}}=1$ is for the standard Newtonian case $(\alpha=0)$. The long dashed line above the horizontal standard line is for $\alpha=1$, the dotted line is for $\alpha=1 / 2$ and the dotted-dashed line is for $\alpha=1 / 4$. For negative values of $\alpha$ the lines are below the standard horizontal case. Small dashed line is for $\alpha=-1 / 2$ and double-dotted-dashed line is for $\alpha=-1 / 4$. We should notice that even though $\lambda=5 \mathrm{Mpc} / h, F_{\mathrm{SF}}$ gives an important contribution to the force between particles for $r>\lambda$. 


\section{Results}

In this section, we present results of cosmological simulations of a $\Lambda \mathrm{CDM}$ universe with and without SF contribution in order to study the large-scale structure formation.

We have used the standard Zel'dovich approximation [5] to provide the initial $256^{3}$ particles displacement of a uniform grid and to assign their initial velocities in a $256 h^{-1} \mathrm{Mpc}$ box [49]. In this approximation the comoving and the Lagrangian coordinates are related by

$$
\begin{aligned}
& \mathbf{x}=\mathbf{q}-\alpha_{Z} \sum_{\mathbf{k}} b_{|\mathbf{k}|}(t) \mathbf{S}_{|\mathbf{k}|}(\mathbf{q}) \\
& \mathbf{p}=-\alpha_{Z} a^{2} \sum_{\mathbf{k}} b_{|\mathbf{k}|}(t)\left(\frac{\dot{b}_{|\mathbf{k}|}(t)}{b_{|\mathbf{k}|}(t)}\right) \mathbf{S}_{|\mathbf{k}|}(\mathbf{q}),
\end{aligned}
$$

where the displacement vector $\mathbf{S}$ is related to the velocity potential $\Phi$ and the power spectrum of fluctuations $P(|k|)$ :

$$
\begin{aligned}
\mathbf{S}_{|\mathbf{k}|}(\mathbf{q}) & =\nabla_{q} \Phi_{|\mathbf{k}|}(\mathbf{q}), \\
\Phi_{|\mathbf{k}|} & =\sum_{\mathbf{k}}\left(a_{\mathbf{k}} \cos (\mathbf{k} \cdot \mathbf{q})+b_{\mathbf{k}} \sin (\mathbf{k} \cdot \mathbf{q})\right),
\end{aligned}
$$

where $a$ and $b$ are gaussian random numbers with the mean zero and dispersion $\sigma^{2}=P(k) / k^{4}$,

$$
a_{\mathbf{k}}=\frac{\sqrt{P(|k|)}}{|k|^{2}} G(0,1), \quad b_{\mathbf{k}}=\frac{\sqrt{P(|k|)}}{|k|^{2}} G(0,1),
$$

where $G(0,1)$ is a gaussian number with mean zero and dispersion 1 .

The parameter $\alpha_{Z}$ together with the power spectrum $P(k)$ defines the normalization of the fluctuations. The initial power spectrum was generated using the fitting formula by [50] for the transfer function. This formula is a slight variation of the common BBKS fit [51].

Therefore, for the standard $\Lambda \mathrm{CDM}$ we have for the initial condition that the starting redshift is $z_{\text {in }}=50$ and we choose the following cosmology: $\Omega_{\mathrm{DM}}=0.314$ (where $\Omega_{\mathrm{DM}}$ includes cold dark matter and baryons), $\Omega_{B}=0.044, \Omega_{\Lambda}=0.686$, $H_{0}=71 \mathrm{~km} / \mathrm{s} / \mathrm{Mpc}, \sigma_{8}=0.84$, and $n=0.99$. Particle masses are in the order of $1.0 \times 10^{10} M_{\odot}$. The individual softening length was $50 \mathrm{kpc} / \mathrm{h}$. This choice of softening length is consistent with the mass resolution set by the number of particles. All these values are in concordance with measurements of cosmological parameters derived from the seven-year data of the WMAP [6]. The initial conditioncalled the big box case-is in the Cosmic Data Bank web page: http://t8web.lanl.gov/people/heitmann/test3.html. See [49] for more details.

Because the visible component is the smaller one and given our interest to test the consequences of including an SF contribution to the evolution equations, our model excludes gas particles, but all its mass has been added to the dark matter. We restrict the values of $\alpha$ to the interval $(-1,1)$ (see [38-41]) and use $\lambda=1,5,10,20 \mathrm{Mpc} / h$, since these values sweep the scale lengths present in the simulations.

In all the simulations we have done, with or without the scalar field contribution, we demand that the cosmological model be flat. In this model with a scalar field a flat universe is obtained if $\Omega_{m}^{(\alpha)}+\Omega_{\Lambda}^{(\alpha)}=1$, with $\Omega_{m}^{(\alpha)} \equiv \Omega_{m} /(1+\alpha)$ and $\Omega_{\Lambda}^{(\alpha)} \equiv \Omega_{\Lambda} /(1+\alpha)$. Then, for positive values of $\alpha$ we need a factor $(1+\alpha)$ with more energy content $\left(\Omega_{m}\right.$ and $\left.\Omega_{\Lambda}\right)$ than that in the standard cosmology whereas for negative values of $\alpha$ we need a factor of $(1+\alpha)$ with less $\Omega_{m}$ and $\Omega_{\Lambda}$ to have a flat universe. With this recipe and for a given value of $\alpha$ we modified the above $\Lambda$ CDM initial condition accordingly.

In Figure 3 we show how the above initial conditions evolve and give us the LSS formation process without SF and with SF: in (a) without SF: in (b) with SF: $\alpha=1$ and $\lambda=1 \mathrm{Mpc} / h$. In (c) with SF: $\alpha=1$ and $\lambda=5 \mathrm{Mpc} / h$ in (d) with SF: $\alpha=1$ and $\lambda=10 \mathrm{Mpc} / h$ in (e) with SF: $\alpha=1$ and $\lambda=20 \mathrm{Mpc} / h$ in (f) With SF: $\alpha=-1 / 2$ and $\lambda=5 \mathrm{Mpc} / h$ in (g) With SF: $\alpha=-1 / 4$ and $\lambda=5 \mathrm{Mpc} / h$.

To study the structure formation in the universe we follow the evolution of the overdensity [4],

$$
\delta(\mathbf{x}) \equiv \frac{\rho(\mathbf{x})}{\rho_{0}}-1,
$$

where $\rho_{0}$ is the average density over a volume $V$ and $\mathbf{x}$ is the comoving distance related to the physical density by $\mathbf{r}=$ $a(t) \mathbf{x}$. In the linear regime $\delta \ll 1$.

The correlation function tells us how $\delta$ is correlated in two nearby points $\mathbf{x}^{\prime}$ and $\mathbf{x}^{\prime}+\mathbf{x}$,

$$
\xi(\mathbf{x}) \equiv\left\langle\delta\left(\mathbf{x}^{\prime}\right) \delta\left(\mathbf{x}^{\prime}+\mathbf{x}\right)\right\rangle,
$$

and the power spectrum is the Fourier transform of the correlation function

$$
\xi(x)=\frac{1}{V} \sum_{\mathbf{k}} P(\mathbf{k}) e^{i \mathbf{k} \cdot \mathbf{x}} .
$$

In Figure 4(a) we show the evolution of the power spectrum for the big box $L=256 \mathrm{Mpc} / h$ without SF, for several values of the redshift $z$. We have used the POWMES code to compute the matter power spectrum [52]. In this figure we can appreciate how it is forming structures, solid line is the power spectrum for the present epoch $z=0$. Figure 4(b) shows the power spectrum for the same values as in Figure 3. Continuous line is without SF. Dashed line is with SF: $\alpha=1$ and $\lambda=5 \mathrm{Mpc} / h$. Dashed-dotted line is with SF: $\alpha=-1 / 2$ and $\lambda=5 \mathrm{Mpc} / h$. The curve that is just below the continuous line (dotted line) is with SF: $\alpha=-1 / 4$ and $\lambda=5 \mathrm{Mpc} / h$. More greater values of the power spectrum means more structure formation. Therefore, the inclusion of an SF modifies the structure formation process. Depending on the values of its parameters, $\alpha$ and $\lambda$, we can obtain more structure or less structure at the present epoch, $z=0$.

In our results as shown in Figures 3(c), 3(f), and 3(g), and Figure 4 we have used a fix value of $\lambda=5 \mathrm{Mpc} / h$. For a given $\lambda$ the role of SF parameter $\alpha$ on the structure formation can be inferred by looking at (59) and (60) and Figure 2 where we show the behavior of $F_{\mathrm{SF}}$ as a function of distance for several values of $\alpha$. The factor $F_{\mathrm{SF}}$ augments (diminishes) for positive (negative) values of $\alpha$ for small distances compared to $\lambda$, resulting in more (less) structure formation for positive (negative) values of $\alpha$ compared 


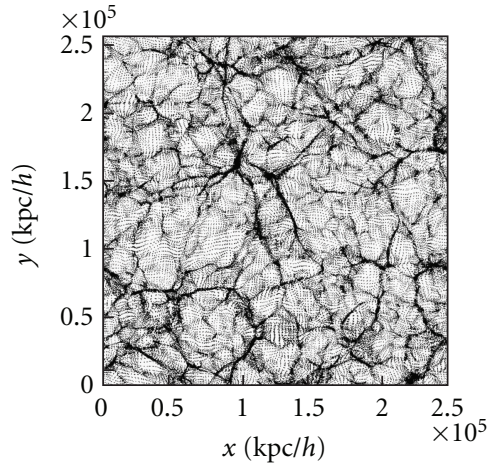

(a) Without SF

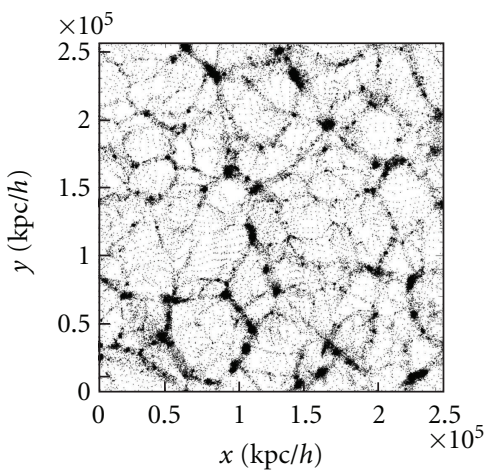

(d) With SF: $\alpha=1, \lambda=10 \mathrm{Mpc} / \mathrm{h}$

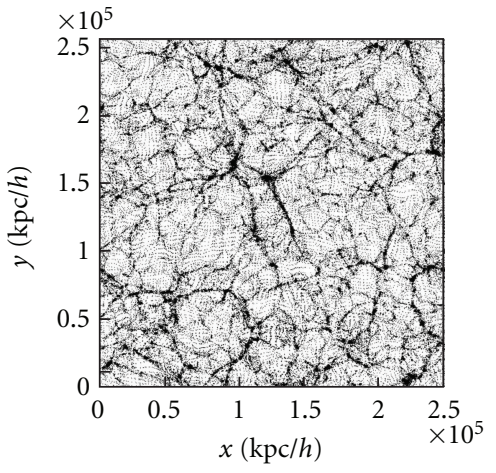

(b) With SF: $\alpha=1, \lambda=1 \mathrm{Mpc} / \mathrm{h}$

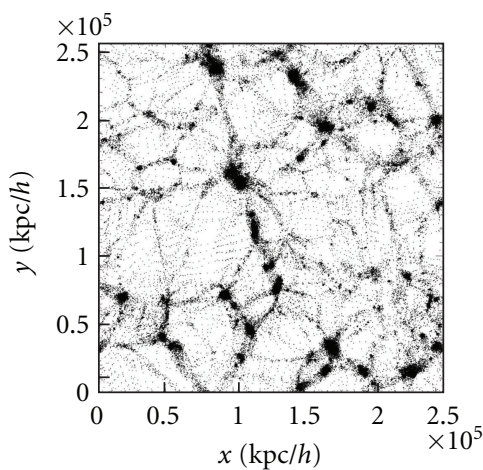

(e) With SF: $\alpha=1, \lambda=20 \mathrm{Mpc} / \mathrm{h}$

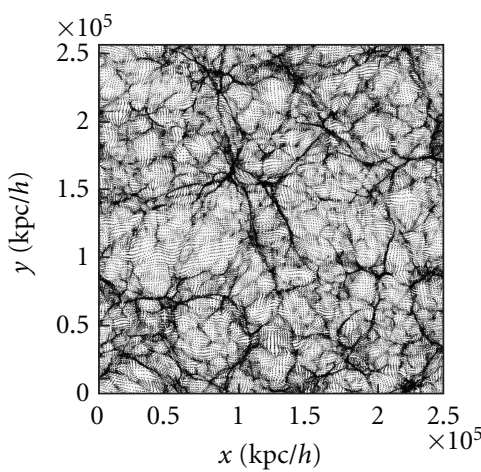

(g) With SF: $\alpha=-1 / 4, \lambda=5 \mathrm{Mpc} / \mathrm{h}$

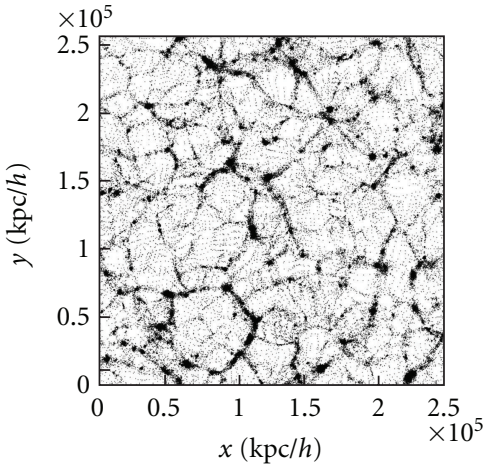

(c) With SF: $\alpha=1, \lambda=5 \mathrm{Mpc} / \mathrm{h}$

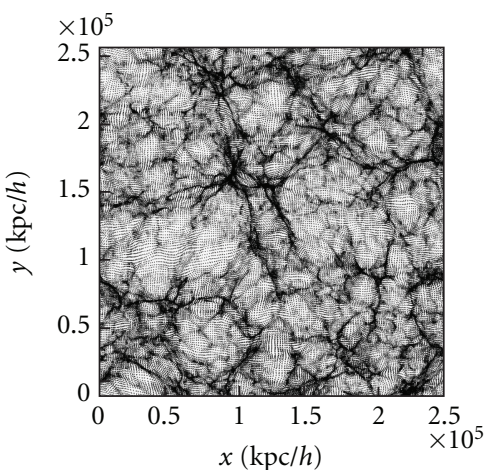

(f) With SF: $\alpha=-1 / 2, \lambda=5 \mathrm{Mpc} / \mathrm{h}$

FIGURE 3: (a) LSS formation process without the SF contribution. (b) and (c) LSS formation process with the SF contribution. (b) $\alpha=1$ and $\lambda=1 \mathrm{Mpc} / h$. (c) $\alpha=1$ and $\lambda=5 \mathrm{Mpc} / h$. (d) and (e) LSS formation process with the SF contribution. (d) $\alpha=1 \mathrm{and} \lambda=10 \mathrm{Mpc} / \mathrm{h}$. (e) $\alpha=1$ and $\lambda=20 \mathrm{Mpc} / h$. (f) and (g) LSS formation process with the SF contribution. (f) $\alpha=-1 / 2$ and $\lambda=5 \mathrm{Mpc} / h$. (g) $\alpha=-1 / 4$ and $\lambda=5 \mathrm{Mpc} / h$.

to the $\Lambda \mathrm{CDM}$ model. In the case of the upper curve in Figure 4(b), for $r \ll \lambda$, the effective gravitational pull has been augmented by a factor of 2 , in contrast to the case shown with the lower line in Figure 4(b) where it has been diminished by a factor of $1 / 2$. That is why we observe for $r<\lambda$ more structure formation in the case of the upper curve in Figure 4(b) and lesser in the case of the lower curve in the same figure. The effect is then, for an increasing positive $\alpha$, to speed up the growth of perturbations, then the halos, and then the clusters, whereas negative values of $\alpha(\alpha \rightarrow-1)$ tend to slow down the growth. We also observe that for the large-scale regimen of our simulations $(k<0.1 h / \mathrm{Mpc})$ they tend to predict almost the same structure formation. From comparison with the experimental results, we see that the $\Lambda$ CDM agrees well with SDSS observations, but predicts more structure formation than observations show in the Lyman- $\alpha$ forest power spectrum. In general the more favored model is the model with SF with $\alpha=-1 / 2$ and $\lambda=5 \mathrm{Mpc} / h$. 


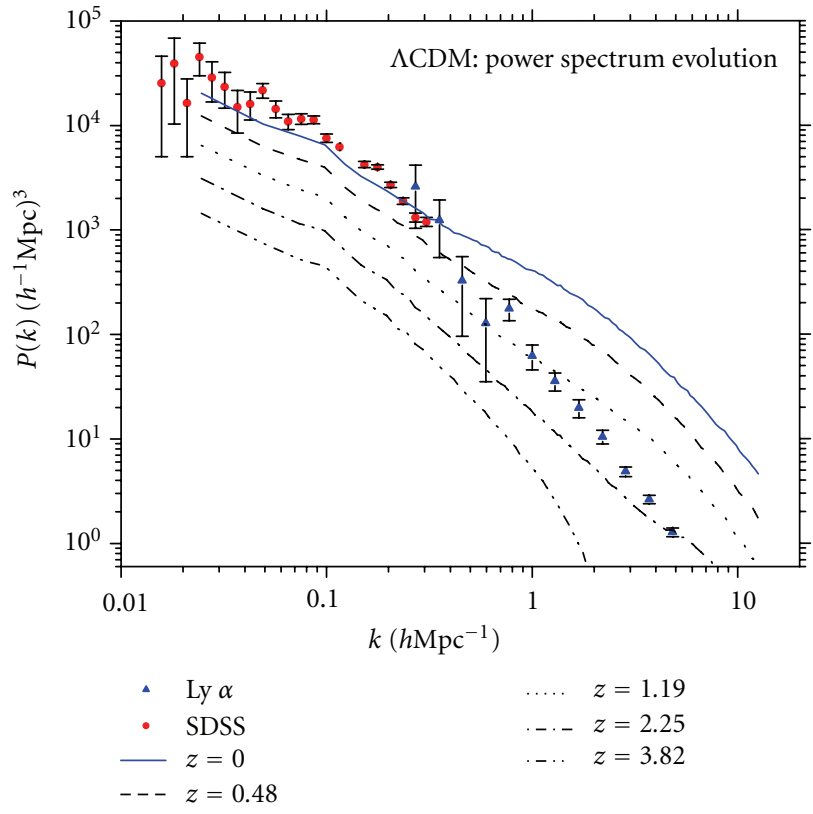

(a)

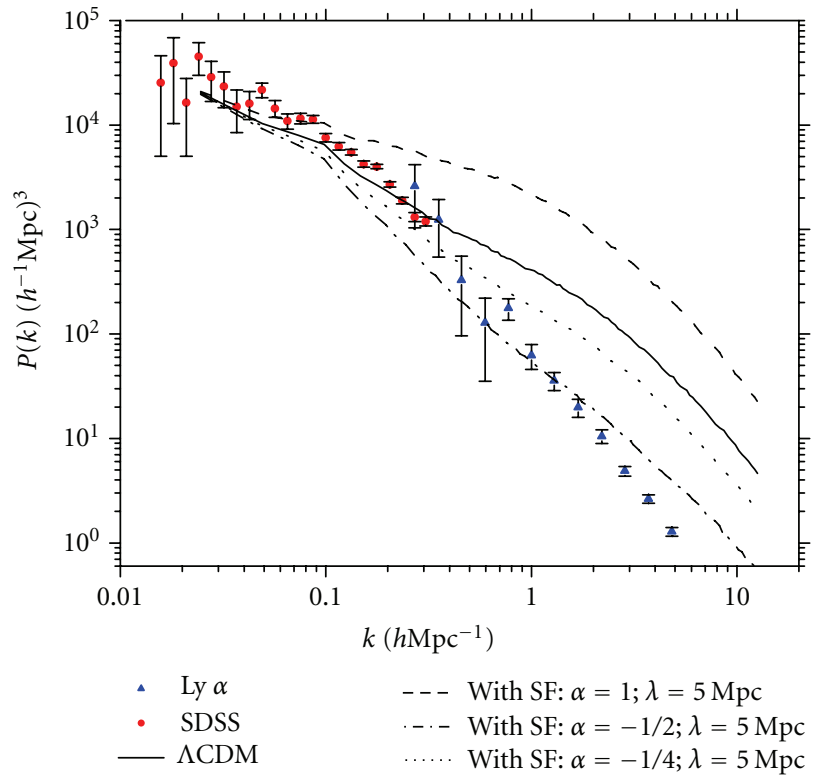

(b)

FIGURE 4: (a) Evolution of the power spectrum for the case which there is no SF. (b) The power spectrum including SF for several values of parameter $\alpha$ and with $\lambda=5 \mathrm{Mpc} / \mathrm{h}$. Experimental data are from galaxies in the Sloan Digital Sky Survey (SDSS) catalog and from an analysis of the Lyman- $\alpha$ forest [54].

\section{Conclusions and Final Comments}

We have used a general, static STT, that is compatible with local observations by the appropriate definition of the background field constant, that is, $\langle\phi\rangle=(1+\alpha) / G_{N}$, to study the LSS formation process. The initial condition for the several cases (different values of parameter $\alpha$ ) was built in such a way that the geometry of the model universe was flat. Quantitatively, this demands that our models have $\Omega /(1+\alpha)=1$ and this changes the amount of dark matter and energy of the models in order to have a flat cosmology.

Using the resulting modified dynamical equations, we have studied the LSS formation process of a $\Lambda$ CDM universe. We varied the amplitude and sign of the strength of the SF (parameter $\alpha$ ) in the interval $(-1,1)$ and performed several 3D simulations with the same initial conditions. From our simulations we have found that the inclusion of the SF changes the local dynamical properties of the most massive groups however, the overall structure is very similar, as can be seen in Figure 3.

The general gravitational effect is that the interaction between dark matter particles given by the potential $\Phi_{N}$ (see (15)) changes by a factor $F_{\mathrm{SF}}$, (see (60)), in comparison with the purely Newtonian case. Thus, for $\alpha>0$ the growth of structures speeds up in comparison with the Newtonian case (without SF). For the $\alpha<0$ case the effect is to diminish the formation of structures.

It is important to note that particles in our model are gravitating particles and that the SF acts as a mechanism that modifies gravity. The effective mass of the SF $\left(m_{\mathrm{SF}}=1 / \lambda\right)$ only sets an interaction length scale for the Yukawa term.
In this work we only varied the amplitude of the SFparameter $\alpha$-leaving the scale length, $\lambda$, of the SF unchanged. However, in other studies we have done [47] we have found that increasing $\lambda$ enhances the structure formation process for $\alpha$ positive and decreasing $\lambda$ makes the structures grow at a slower rate.

We have computed the mass power spectrum in order to study the LSS formation process. The theoretical scheme we have used is compatible with local observations because we have defined the background field constant $\langle\phi\rangle=G_{N}^{-1}(1+$ $\alpha)$ or equivalently that the local gravitational constant is given by $(1+\alpha)\langle\phi\rangle^{-1}$, instead of being given by $\langle\phi\rangle^{-1}$. A direct consequence of the approach is that the amount of matter (energy) has to be increased for positive values of $\alpha$ and diminished for negative values of $\alpha$ with respect to the standard $\Lambda \mathrm{CDM}$ model in order to have a flat cosmological model. Quantitatively, our model demands to have $\Omega /(1+\alpha)=1$ and this changes the amount of dark matter and energy of the model for a flat cosmological model, as assumed. The general gravitational effect is that the interaction including the SF changes by a factor $F_{\mathrm{SF}}(r, \alpha, \lambda) \approx$ $1+\alpha(1+(r / \lambda))$ for $r<\lambda$ in comparison with the Newtonian case. Thus, for $\alpha>0$ the growth of structures speeds up in comparison with the Newtonian case. For the $\alpha<0$ case the effect is to diminish the formation of structures. For $r>\lambda$ the dynamics is essentially Newtonian.

Comparison of the power spectrums from galaxies in the SDSS catalog versus those inferred from Lyman- $\alpha$ forest observations tells us that $\Lambda \mathrm{CDM}$ predicts more structure formation in the regime of smaller scales $(k>0.4 \mathrm{~h} / \mathrm{Mpc})$ whereas the model with SF with $\alpha=-1 / 2$ and $\lambda=5 \mathrm{Mpc} / \mathrm{h}$ 
follows the general trend of the observed power spectrum. In this way we are able to construct a model that predicts the observed structure formation in the regime of small scales, with lesser number of halo satellites than the $\Lambda \mathrm{CDM}$ model.

\section{References}

[1] N. Bretoon, J. L. Cervantes-Cota, and M. Salgado, Eds., The Early Universe and Observational Cosmology, Springer, Berlin, Germany, 2004.

[2] J. F. Navarro, C. S. Frenk, and S. D. M. White, "The structure of cold dark matter halos," Astrophysical Journal Letters, vol. 462, no. 2, pp. 563-575, 1996.

[3] J. F. Navarro, C. S. Frenk, and S. D. M. White, "A universal density profile from hierarchical clustering," Astrophysical Journal Letters, vol. 490, no. 2, pp. 493-508, 1997.

[4] J. Binney and S. Tremaine, Galactic Dynamics, Princeton University Press, Princeton, NJ, USA, 2008.

[5] Y. B. Zeldovich, "Gravitational instability: an approximate theory for large density perturbations," Astronomy and Astrophysics, vol. 5, pp. 84-89, 1970.

[6] E. Komatsu, K. M. Smith, J. Dunkley et al., "Seven-year wilkinson microwave anisotropy probe (WMAP) observations: cosmological interpretation," The Astrophysical Journal, vol. 192, no. 2, p. 18, 2011.

[7] A. G. Riess, A. V. Filippenko, P. Challis et al., "Observational evidence from supernovae for an accelerating universe and a cosmological constant," Astronomical Journal, vol. 116, no. 3, pp. 1009-1038, 1998.

[8] S. Perlmutter, G. Aldering, G. Goldhaber et al., "Measurements of $\Omega$ and $\Lambda$ from 42 high-redshift Supernovae," Astrophysical Journal Letters, vol. 517, no. 2, pp. 565-586, 1999.

[9] B. Ratra and P. J. E. Peebles, "Cosmological consequences of a rolling homogeneous scalar field," Physical Review D, vol. 37, no. 12 , pp. 3406-3427, 1988.

[10] C. Wetterich, "Cosmology and the fate of dilatation symmetry," Nuclear Physics B, vol. 302, no. 4, pp. 668-696, 1988.

[11] R. R. Caldwell, "A phantom menace? Cosmological consequences of a dark energy component with super-negative equation of state," Physics Letters B, vol. 545, no. 1-2, pp. 2329, 2002.

[12] R. R. Caldwell, M. Kamonkowski, N. N. Weinberg et al., "Phantom energy: dark energy with $w<-1$ causes a cosmic doomsday," Physical Review Letters, vol. 91, Article ID 071301, 4 pages, 2003.

[13] B. Feng, X. L. Wang, and X. M. Zhang, "Dark energy constraints from the cosmic age and supernova," Physics Letters $B$, vol. 607, no. 1-2, pp. 35-41, 2005.

[14] Y. F. Cai, E. N. Saridakis, M. R. Setare, and J. Q. Xia, "Quintom cosmology: theoretical implications and observations," Physics Reports, vol. 493, no. 1, pp. 1-60, 2010.

[15] B. Li and J. D. Barrow, "N-body simulations for coupled scalar-field cosmology," Physical Review D, vol. 83, Article ID 024007, 25 pages, 2011.

[16] B. Li, D. F. Mota, and J. D. Barrow, "N-body simulations for extended quintessence models," The Astrophysical Journal, vol. 728, no. 2, p. 109, 2011.

[17] M. A. Rodriguez-Meza, J. Klapp, J. L. Cervantes-Cota, and H. Dehnen, "The influence of scalar fields in protogalactic interactions," in Exact Solutions and Scalar Fields in Gravity: Recent Developments, A. Macias, J. L. Cervantes-Cota, and C. Lammerzahl, Eds., p. 213, Kluwer Academic, New York, NY, USA, 2001.
[18] R. Barrena, A. Biviano, M. Ramella, E. E. Falco, and S. Steitz, "The dynamical status of the cluster of galaxies 1E0657-56," Astronomy and Astrophysics, vol. 386, pp. 816-828, 2002.

[19] M. Markevitch, A. H. Gonzalez, L. David et al., "A textbook example of a bow shock in the merging galaxy cluster 1E 065756," Astrophysical Journal Letters, vol. 567, no. 1, pp. L27-L31, 2002.

[20] M. Markevitch, “Pro. X-Ray Universe 2005,” ESA SP-604, Noordwijk, The Netherlands, ESA, 2006, Edited by Wilson.

[21] D. Clowe, A. Gonzalez, and M. Markevitch, "Weak-lensing mass reconstruction of the interacting cluster 1E 0657-558: direct evidence for the existence of dark matter," Astrophysical Journal, vol. 604, no. 2, pp. 596-603, 2004.

[22] D. Clowe, M. Bradač, A. H. Gonzalez et al., "A direct empirical proof of the existence of dark matter," Astrophysical Journal, vol. 648, no. 2, pp. L109-L113, 2006.

[23] C. Mastropietro and A. Burkert, "Simulating the bullet cluster," Monthly Notices of the Royal Astronomical Society, vol. 389, no. 2, pp. 967-988, 2008.

[24] M. Milosavljevic, J. Koda, D. Nagai et al., “The cluster-merger shock in 1E 0657-56: faster than a speeding bullet?" The Astrophysical Journal Letters, vol. 661, no. 2, p. L131, 2007.

[25] V. Springel and G. R. Farrar, "The speed of the "bullet" in the merging galaxy cluster 1E0657-56," Monthly Notices of the Royal Astronomical Society, vol. 380, no. 3, pp. 911-925, 2007.

[26] M. Takizawa, "Hydrodynamic simulations of a moving substructure in a cluster of galaxies: cold fronts and turbulence generation," Astrophysical Journal Letters, vol. 629, no. 2, pp. 791-796, 2005.

[27] M. Takizawa, "On the X-ray and mass distribution in the merging galaxy cluster 1E 0657-56: ram pressure-stripping in substructures with an NFW density profile," Publications of the Astronomical Society of Japan, vol. 58, no. 6, pp. 925-929, 2006.

[28] L. Lee and E. Komatsu, "Bullet cluster: a challenge to LCDM cosmology," The Astrophysical Journal, vol. 718, no. 1, pp. 6065, 2010, http://arxiv.org/abs/1003.0939.

[29] V. Faraoni, Cosmology in Scalar-Tensor Gravity, Kluwer Academic, Dordrecht, The Netherlands, 2004.

[30] M. A. Rodríguez-Meza and J. L. Cervantes-Cota, "Potentialdensity pairs for spherical galaxies and bulges:the influence of scalar fields," Monthly Notices of the Royal Astronomical Society, vol. 350, no. 2, pp. 671-678, 2004.

[31] P. J. E. Peebles, The Large-Scale Structure of the Universe, Princeton University Press, Princeton, NJ, USA, 1980.

[32] T. Helbig, "Gravitational effects of light scalar particles," Astrophysical Journal Letters, vol. 382, no. 1, pp. 223-232, 1991.

[33] L. O. Pimentel and O. Obregón, "A scalar-tensor theory and the new interaction," Astrophysics and Space Science, vol. 126, no. 2, pp. 231-234, 1986.

[34] M. Salgado, "On the linear and weak-field limits of scalartensor theories of gravity," http://arxiv.org/abs/gr-qc/0202082.

[35] M. A. Rodríguez-Meza, J. L. Cervantes-Cota, M. I. Pedraza, J. F. Tlapanco, and E. M. D. L. Calleja, "Potential-density pairs for axisymmetric galaxies: the influence of scalar fields," General Relativity and Gravitation, vol. 37, no. 4, pp. 823-829, 2005.

[36] E. Fischbach and C. L. Talmadge, The Search for NonNewtonian Gravity, Springer, New York, NY, USA, 1999.

[37] J. D. Jackson, Classical Electrodynamics, John Wiley \& Sons, New York, NY, USA, 2nd edition, 1975.

[38] R. Nagata, T. Chiba, and N. Sugiyama, "Observational consequences of the evolution of primordial fluctuations in scalartensor cosmology," Physical Review D, vol. 66, no. 10, Article ID 103510, 2002. 
[39] R. Nagata, T. Chiba, and N. Sugiyama, "WMAP constraints on scalar-tensor cosmology and the variation of the gravitational constant," Physical Review D, vol. 69, no. 8, Article ID 083512, 2004.

[40] A. Shirata, T. Shiromizu, N. Yoshida, and Y. Suto, "Galaxy clustering constraints on deviations from Newtonian gravity at cosmological scales," Physical Review D, vol. 71, no. 6, Article ID 064030, 8 pages, 2005.

[41] K. I. Umezu, K. Ichiki, and M. Yahiro, "Cosmological constraints on Newton's constant," Physical Review D, vol. 72, no. 4, Article ID 044010, 5 pages, 2005.

[42] E. Bertschinger, "Simulations of structure formation in the universe," Annual Review of Astronomy and Astrophysics, vol. 36, no. 1, pp. 599-654, 1998.

[43] L. Hernquist, F. R. Bouchet, and Y. Suto, "Application of the Ewald method to cosmological N-body simulations," Astrophysical Journal, Supplement, vol. 75, no. 2, pp. 231-240, 1991.

[44] M. A. Rodríguez-Meza, A. X. González-Morales, R. F. Gabbasov, and J. L. Cervantes-Cota, "Cosmological simulations using a static scalar-tensor theory," Journal of Physics, vol. 91, Article ID 012012, 2007.

[45] M. A. Rodríguez-Meza, "Scalar-tensor cosmological simulations," in Proceedings of the 3rd Mexican Meeting on Mathematical and Experimental Physics, pp. 302-309, September 2007.

[46] M. A. Rodríguez-Meza, "Cosmological simulations of the Santa Barbara cluster: the influence of scalar fields," in Proceedings of the 3rd International Meeting on Gravitation and Cosmology, vol. 1083 of AIP Conference Proceedings, pp. 190200, Morelia, Mexico, 2009.

[47] M. A. Rodríguez-Meza, "Cosmological simulations: the role of scalar fields," in Proceedings of the XIII Mexican School of Particles and Fields, vol. 1116 of AIP Conference Proceedings, pp. 171-178, 2009.

[48] M. A. Rodríguez-Meza, "Power spectrum of large-scale structure cosmological models in the framework of scalar-tensor theories," Journal of Physics, vol. 229, no. 1, Article ID 012063, 2010.

[49] K. Heitmann, P. M. Ricker, M. S. Warren, and S. Habib, "Robustness of cosmological simulations. I. Large-scale structure," Astrophysical Journal, Supplement, vol. 160, no. 1, pp. 28-58, 2005.

[50] A. Klypin and J. Holtzman, "Particle-Mesh code for cosmological simulations," http://arxiv.org/abs/astro-ph/9712217.

[51] J. M. Bardeen, J. R. Bond, N. Kaiser, and A. S. Szalay, "The statistics of peaks of Gaussian random fields," Astrophysical Journal, vol. 304, pp. 15-61, 1986.

[52] S. Colombi, A. Jaffe, D. Novikov, and C. Pichon, "Accurate estimators of power spectra in N-body simulations," Monthly Notices of the Royal Astronomical Society, vol. 393, no. 2, pp. 511526, 2009.

[53] D. N. Spergel, L. Verde, H. V. Peiris et al., "First-year wilkinson microwave anisotropy probe (WMAP) observations: determination of cosmological parameters," Astrophysical Journal, Supplement, vol. 148, no. 1, pp. 175-194, 2003.

[54] M. Tegmark, M. R. Blanton, M. A. Strauss et al., "The threedimensional power spectrum of galaxies from the sloan digital sky survey," Astrophysical Journal, vol. 606, no. 2, pp. 702-740, 2004. 

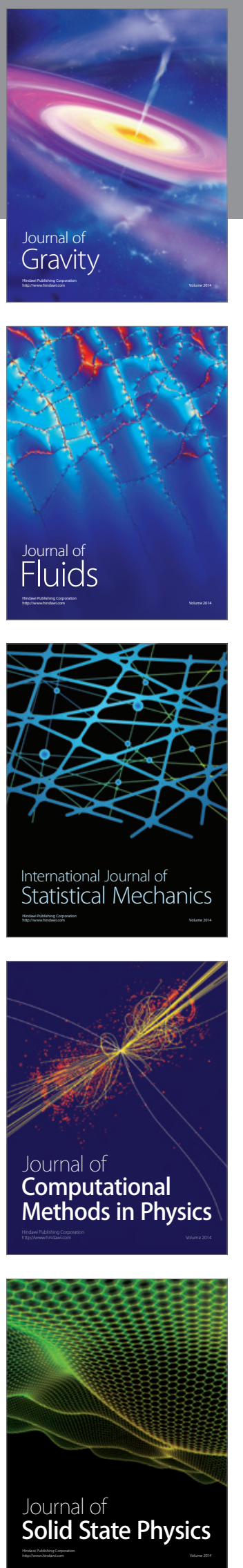

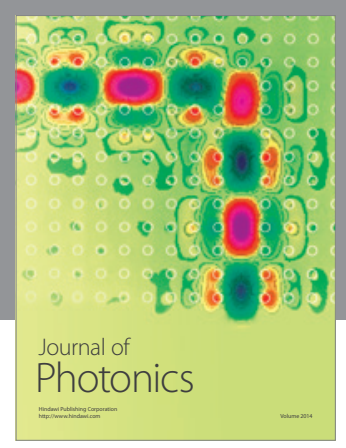

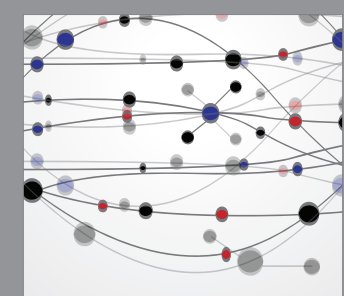

The Scientific World Journal
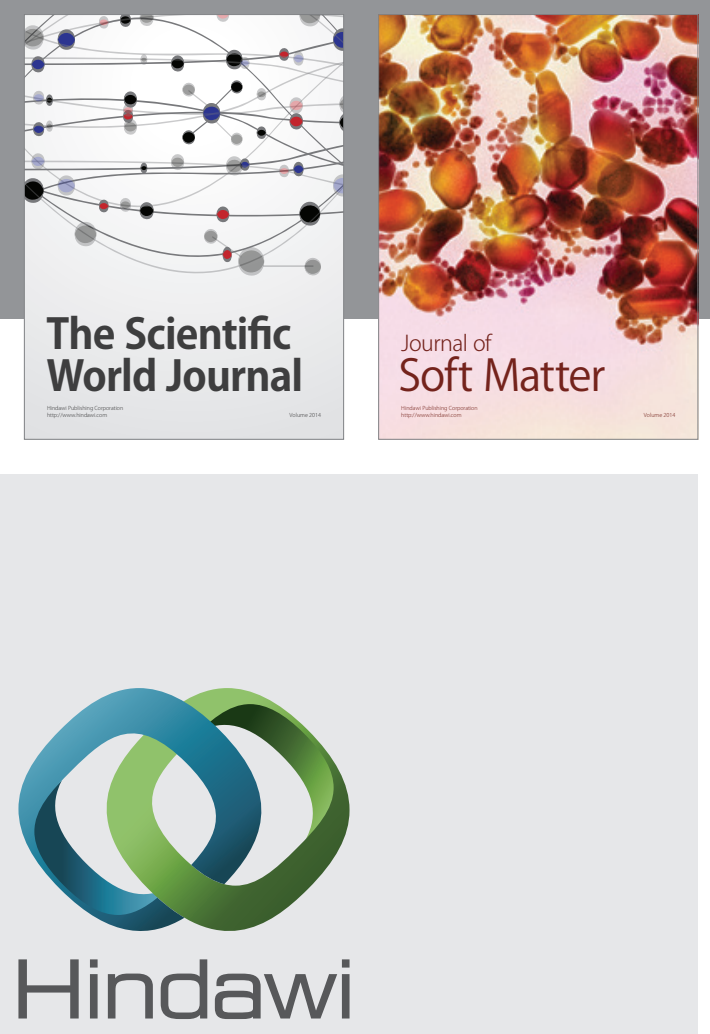

Submit your manuscripts at

http://www.hindawi.com
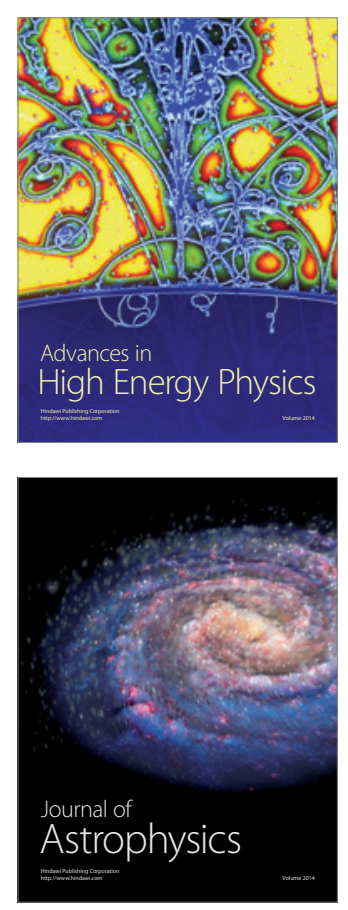
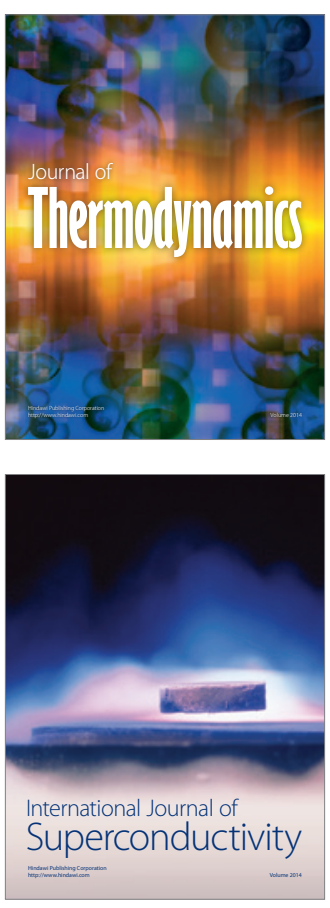
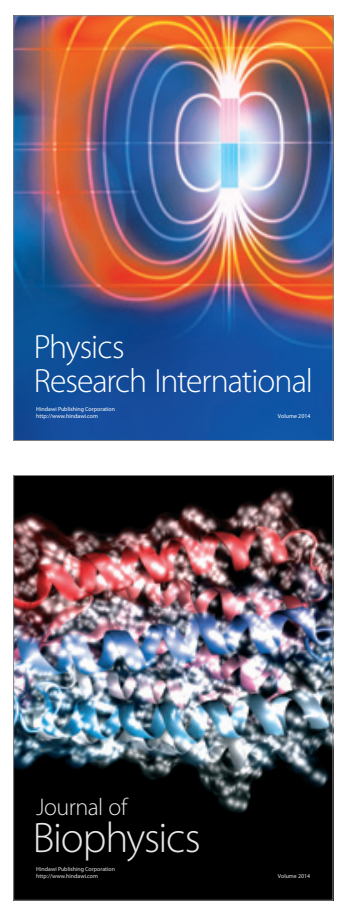
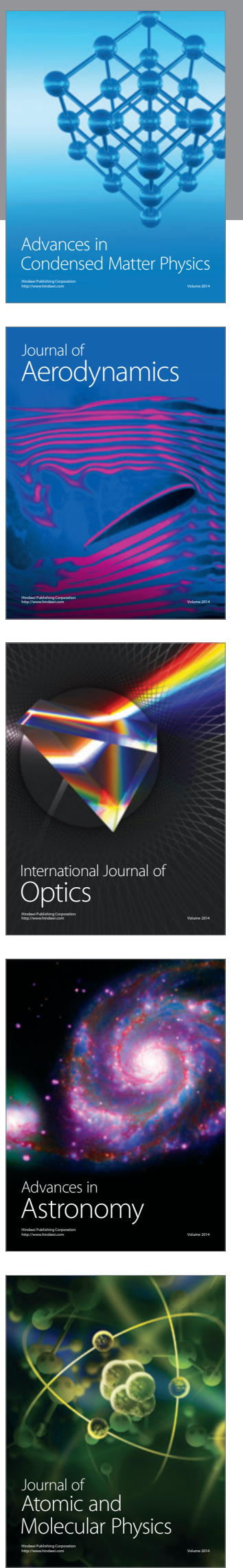